\title{
Do X-ray plasma ejections accelerate Coronal Mass Ejections?
}

\author{
M. Tomczak ${ }^{\star}$
}

Astronomical Institute, University of Wrocław, ul. Kopernika 11, 51-622 Wrocław, Poland

Received 6 January 2003 / Accepted 15 December 2003

\begin{abstract}
The X1.2 flare occurred on 23 April 1998 approximately $13^{\circ}$ behind the eastern solar limb. Its C2.0 precursor is identified as a rapidly expanding system of magnetic loops that was well-observed by Yohkoh. Basic parameters of this ejection are estimated based on images from the SXT telescope. The evolution of the X-ray ejection is compared to a sequence of CME images obtained by the LASCO coronagraph onboard SOHO. Evidence that the X-ray ejection is responsible for an additional acceleration of the $\mathrm{CME}$ is presented. A possible scenario of the event is suggested.
\end{abstract}

Key words. Sun: corona - flares - coronal mass ejections (CMEs) - X-rays, gamma rays

\section{Introduction}

It has been generally accepted that coronal mass ejections (CMEs) and flares are two different manifestations of the same magnetic process in the solar corona. The relationship between CMEs and flares has been a subject of comprehensive studies for many years.

The most common classification scheme of CMEs includes two principal types: flare-associated and non-flare-associated (e.g. MacQueen \& Fisher 1983; Kahler 1992; Sheeley et al. 1999; St.Cyr et al. 2000; Švestka 2001; Andrews \& Howard 2001; Wu et al. 2001; Moon et al. 2002). CMEs of the first, less frequent type are usually brighter, larger and faster $\left(500-1000 \mathrm{~km} \mathrm{~s}^{-1}\right)$ than of the second type $\left(400-600 \mathrm{~km} \mathrm{~s}^{-1}\right)$. They are formed and accelerated lower in the corona and correlate better with type II and type IV radio bursts than the second type. Moreover, flare-associated events decelerate after their initial acceleration, non-flare-associated ones do not. Finally, flare-associated CMEs are more likely to produce shocks at $1 \mathrm{AU}$.

A CME onset is rather poorly understood. Any studies of this phase need to incorporate observations made in other wavebands than the optical range, preferably in UVor X-rays, to search for signatures of a CME onset. For flare-associated events the chronology excludes flares as CME drivers. However, very often the time of the projected onset of a CME agrees with the occurrence of a flare precursor (Harrison 1986).

The Japanese satellite Yohkoh offered new possibilities to investigate in $\mathrm{X}$-rays the direct signatures of a CME onset. Among such signatures there are flare-associated ejections of hot plasma, which are frequently observed in the Soft X-ray Telescope (SXT) images (Shibata et al. 1995; Tsuneta 1997; Ohyama \& Shibata 1997, 1998; Kundu et al. 2001). This ejected, X-ray emitting plasma (X-ray ejection) usually takes

\footnotetext{
* e-mail: tomczak@astro.uni.wroc.pl
}

the form of a blob-like feature (plasmoid) or a loop system. Its acceleration to apparent velocities of $50-500 \mathrm{~km} \mathrm{~s}^{-1}$ occurs just before or at about the onset of the impulsive phase of the flare.

The first attempt at correlating the X-ray ejections with the CMEs was made by Nitta \& Akiyama (1999). They found that if no CME was detected around the flare time, the flare was not accompanied by an X-ray ejection. They also found that for the majority of events, the estimated CME onset time was close to the time of the X-ray ejection. However, they conclude that "the role of the plasma ejection in a CME is not clear and should be investigated in more detailed studies."

$\mathrm{X}$-ray ejections are distinctly fainter and located somewhat higher in the corona than flares. On the other hand, the exposure time and the position of the field of view of the SXT images are automatically adjusted based on the location and the signal of the brightest pixel. In consequence, $\mathrm{X}$-ray ejections have poor statistics and are located close to the borders of images. Thus, it is difficult to make a detailed analysis of the X-ray ejections without a special treatment.

In this paper a behind-the-limb flare has been chosen. Due to the previous expansion, the flare came into view from behind the solar limb but its occurrence was preceded by an $\mathrm{X}$-ray ejection which expanded faster. In consequence, for several minutes the X-ray ejection was seen on SXT images alone, without the presence of the brighter flare. The analysis of the $\mathrm{X}$-ray ejection is presented in Sect. 2.1 and the evolution of the corresponding CME is shown in Sect. 2.2. The results are discussed in Sect. 3 and a possible scenario of the whole event is suggested in Sect. 4.

\section{Observations}

\subsection{X-ray ejection}

The GOES class X1.2 flare of 23 April 1998 occurred in NOAA Active Region 8210 about $13^{\circ}$ behind the eastern solar limb 


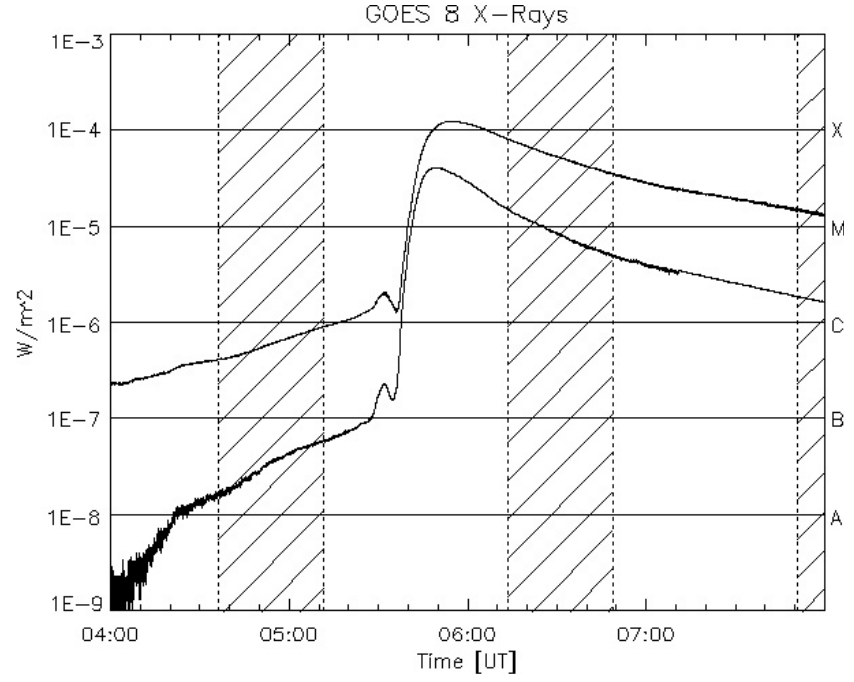

Fig. 1. The GOES light curves for the X1.2 flare of 23 April 1998. Its $\mathrm{C} 2.0$ precursor at about 05:32 UT is clearly seen. The upper curve shows the emission in the 1-8 $\AA$ band, the lower curve shows the emission in the 0.5-4 $\AA$ band. Time intervals shown by the hatched areas indicate times when Yohkoh data were unavailable because of satellite night-time.

(Sato 2001). This flare was preceded by a GOES class C2.0 precursor which had its maximum at about 05:32 UT (Fig. 1). Both events (the flare and its precursor) were well-observed by the Yohkoh satellite in the quiet mode and the flare mode before and after 05:29:20 UT, respectively.

Some light curves made in the higher energies (the Bragg Crystal Spectrometer, BCS, - four channels; the Hard X-ray Telescope, HXT, - channel L) also show the presence of this precursor (Fig. 2). However, some differences are seen depending on energy. First, the maximum time is systematically earlier for the higher energies. Second, an additional peak is visible about 05:29:30 UT in the HXT(L) channel and in the BCS Fe XXV and Fe XXVI channels. Third, in these channels an increase of signal above the background begins about 05:28 UT, whereas at the lower energies (e.g. the BCS Ca XIX and S XV channels) we observe the systematic increase of signal much earlier.

Several soft X-ray images derived by the SXT, illustrating the evolution of the NOAA AR 8210 before the X1.2 flare, are presented in Fig. 3. We see an extended magnetic structure which protrudes above the eastern solar limb $15^{\circ}-20^{\circ}$ south off the equator. The evolution of two brightest features, $\mathrm{A}$ and $\mathrm{B}$, was very slow in contrast to the rapidly expanding X-ray ejection. The ejection became visible in the image at 05:28:34 UT and in the later images it took the form of a rising system of semicircular loops. The passage of the X-ray ejection has distinctly squeezed feature A but it did not change feature B. After 05:36 UT the X-ray ejection faded. One minute later the top of a flaring arcade appeared outside the solar limb and its emission completely dominated the total active region emission.

Some intensity changes in the area between expanding loops and the solar limb were observed. This suggests a 3D-geometry of the X-ray ejection rather than a $2 \mathrm{D}$-geometry. In the case of a hemishell geometry the bright

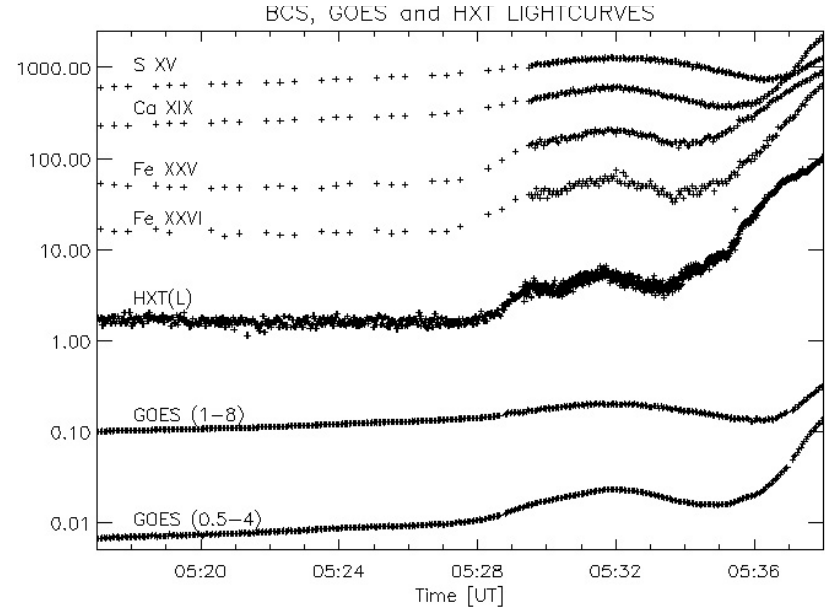

Fig. 2. The light curves of the $\mathrm{C} 2.0$ precursor obtained in four channels of the BCS, in the channel L of the HXT, and in two channels of the GOES are shown. The fluxes are in cts $\mathrm{s}^{-1}, \mathrm{cts} \mathrm{s}^{-1} \mathrm{SC}^{-1}$, and $\mathrm{W} \mathrm{m}^{-2} \times$ $10^{5}$ units, for the BCS, HXT, and GOES, respectively.

loop-like structure is an effect of the accumulation of emission throughout a longer distance in the line-of-sight direction. In this paper the bright boundary of the hemishell will be called the front and the volume below the front - the interior (see Fig. 3).

To examine the suggestion of a 3D-geometry of the X-ray ejection, we estimated the intensity ratio normalized per pixel, $R$, between the front and the interior of the hemishell. We assumed the hemishell thickness to be equal to the height difference, $h_{1}-h_{2}$, between the leading and the trailing edge of the front. We assumed also that the hemishell is filled uniformly with the X-ray emitting plasma. According to the following formula

$R=\sqrt{\frac{h_{1} / h_{2}+1}{h_{1} / h_{2}-1}}$,

the calculated values of the intensity ratio were in the range 2.3-2.7 during the X-ray ejection evolution. The same parameter obtained from the SXT images was in the range 1.4-1.6.

The difference between the values of the ratio $R$ for the hemishell model and for the observations may suggest that the actual thickness of the hemishell was greater than the thickness of the bright boundary observed in the SXT images (see Eq. (1), in which an increment of $h_{1} / h_{2}$ causes a drop of $R$ ). An alternative explanation of this difference are inhomogeneities in the distribution of the X-ray emitting plasma inside the hemishell.

In Fig. 4 the light curves of individual features seen in Fig. 3 are presented. The boundaries of the features were determined as follows: for feature A as the place where the intensity is equal to $30 \%$ of the maximum intensity in the feature, for the $\mathrm{X}$-ray ejection as the place between the leading edge and the solar limb. Feature B fell within the latter boundary, hence its contribution to the light curve of the X-ray ejection is included. Intensity changes of the extended magnetic structure above the $\mathrm{X}$-ray ejection and feature A are described by the light curve called the remainder. It is the result of the subtraction of the 


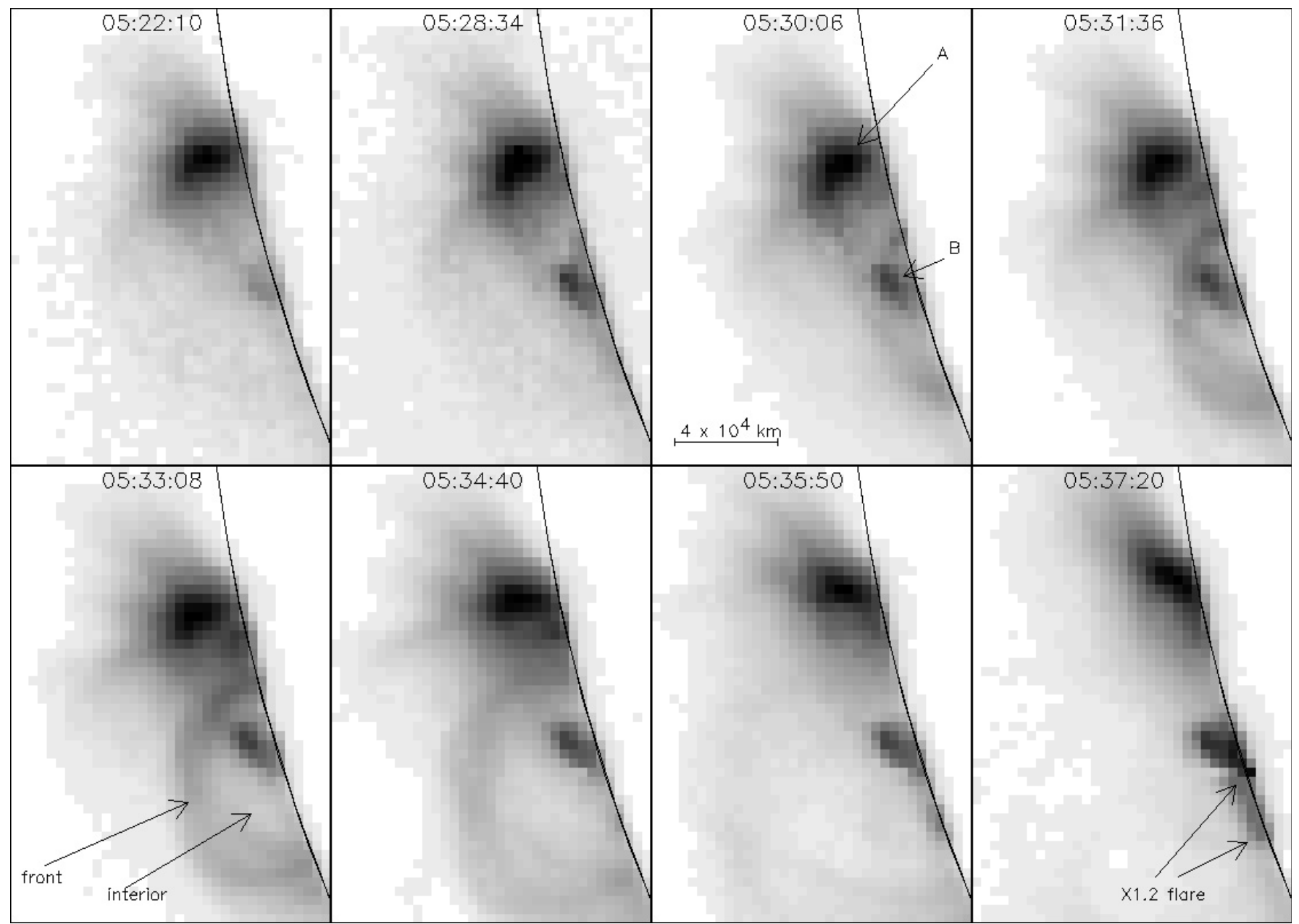

Fig. 3. A sequence of SXT HN AlMg images illustrating the evolution of the X-ray ejection. The continuous curve represents the solar eastern limb. Solar north is up, east to the left.

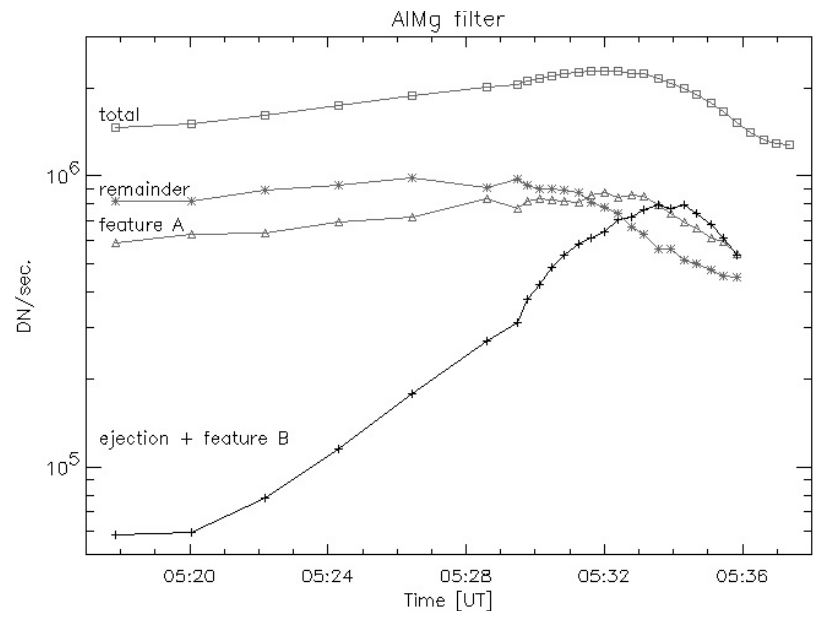

Fig. 4. The SXT(AlMg) light curves of individual features seen in Fig. 3. See text for explanation.

intensities of the X-ray ejection and of features A and B from the light curve of the total images.

The presence of the maximum in the total light curve (Fig. 4) and in the light curves seen in Fig. 2 proves that the
C2.0 precursor occurred in the NOAA AR 8210. The comparison between light curves in Fig. 4 allows us to identify the precursor as the X-ray ejection. Other parts of this active region (the light curves feature $A$ and remainder) showed only a slow, continuous increase of intensity followed by a steeper drop. The comparison between Figs. 3 and 4 shows that the intensity drop began in a particular part of the active region 2-3 min before the X-ray ejection front reached this place. This may suggest the presence of a perturbation accompanying the X-ray ejection which preceded the X-ray ejection front.

In Fig. 4 the maximum of the total light curve occurs about two minutes earlier than the maximum of the X-ray ejection brightness. This difference can be explained recalling that two processes had an influence on the time profile of the precursor. First, the increase of emission from recently heated plasma in the X-ray ejection. Second, the decrease of emission as a result of partial removing of plasma from the magnetic structures which were present in the active region earlier, caused by the perturbation accompanying the X-ray ejection.

To determine more detailed properties of the investigated X-ray ejection, its dynamics has been studied first. Time changes of the maximal height above the chromosphere for the 


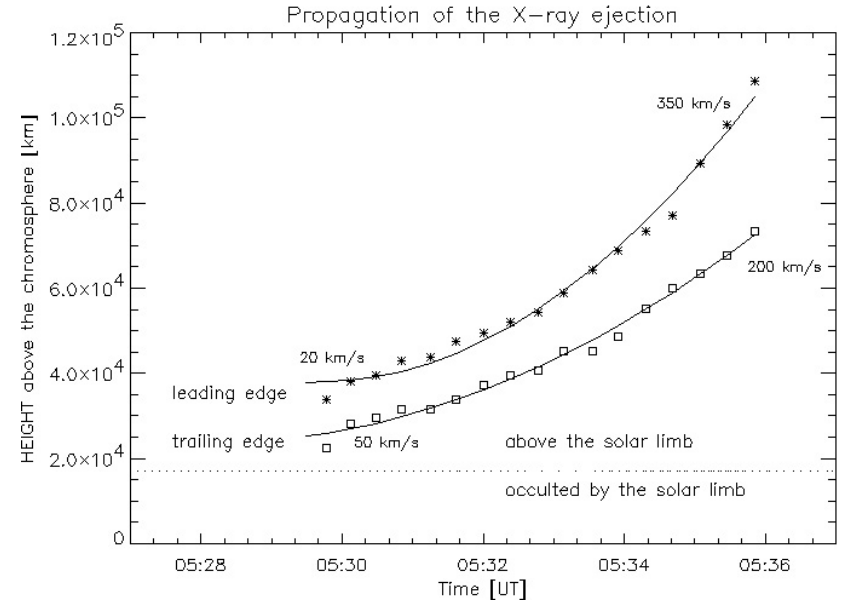

Fig. 5. A plot representing the expansion of the leading (stars) and the trailing edge (boxes) of the X-ray ejection. The least-squares fit to data and example values of velocity are also shown.

leading (stars) and for the trailing edge (squares) of the X-ray ejection front are shown in Fig. 5. The height of the occultation by the solar limb was estimated to be about $1.7 \times 10^{4} \mathrm{~km}$. The best least-square polynomial fit to the data is also plotted. A constant acceleration was found. The obtained values were about $0.95 \mathrm{~km} \mathrm{~s}^{-2}$ and $0.44 \mathrm{~km} \mathrm{~s}^{-2}$ for the leading and the trailing edge of the X-ray ejection front, respectively. The velocity increased from $20 \mathrm{~km} \mathrm{~s}^{-1}$ to $350 \mathrm{~km} \mathrm{~s}^{-1}$ and from $50 \mathrm{~km} \mathrm{~s}^{-1}$ to $200 \mathrm{~km} \mathrm{~s}^{-1}$ for the leading and the trailing edge of the X-ray ejection front, respectively.

In the next step, temperature and emission measure maps were calculated using the SXT observations with filters AlMg and Al12. The only images obtained with these two filters and the pixel size 4 .' $9 \times 4$ ". 9 (so-called Half Normal resolution, $\mathrm{HN}$ ) had a field of view large enough to include the whole $\mathrm{X}$-ray ejection. Other available observations include more images with better $(2$ '. $45 \times 2$ '. 45$)$ spatial resolution (so-called Full Normal, FN), but smaller field of view that included only the northern part of the X-ray ejection. The selected images were time-interpolated and their saturated pixels were replaced with the equivalent pixels from the FN images with a shorter exposure time.

In Fig. 6 three representative temperature maps of the NOAA AR 8210 are presented, the first one before the X-ray ejection and the next two during the $\mathrm{X}$-ray ejection. We see two sources where the hottest plasma is concentrated: above feature $\mathrm{A}$ and in the front of the X-ray ejection. The first source was present before the X-ray ejection and during its propagation did not change significantly. The second source underwent dynamical changes reflecting the rapid expansion of the $\mathrm{X}$-ray ejection. Local islands of the higher temperature seen at 05:33:08 UT have a minor statistical importance.

Despite the similar temperature values in both sources (Fig. 6), we suggest that the hot thermal plasma (15-40 MK) registered in the higher-energy bands - HXT(L), BCS(Fe XXVI), and BCS(Fe XXV) in Fig. 2 - came from the front of the X-ray ejection. First, the broad-band SXT filters are not very sensitive to the hot plasma (e.g. Jakimiec et al. 1998). Second, the source above feature A was present before 05:28 UT when no emission in the higher-energy bands was observed. This kind of emission was detectable after 05:28 UT when the X-ray ejection became visible.

The hot plasma distribution obtained from the SXT images agrees with data from other Yohkoh instruments. Temperature values obtained as from the fit to Ca XIX and S XV spectra are typical (10-12 MK), whereas Fe XXV spectra showed very high (30-40 MK) temperatures. Thus, the Fe XXV spectra, which indicate a hotter plasma, recorded the very hot plasma from the $\mathrm{X}$-ray ejection front. The Ca XIX and S XV spectra registered an emission mix of this hotter plasma and the cooler plasma which was located above feature A.

Several HXT images obtained in channel $\mathrm{L}$ are of rather poor quality because of a low statistic of counts and the motion of the X-ray ejection. They show undersampled or very diffuse hard X-ray sources comprising all of the X-ray ejection with its surrounding area. The recent work by Hudson et al. (2001) confirms that the X-ray ejection can be an evident source of the hard X-ray emission.

A more complete temperature evolution for individual parts of the NOAA AR 8210 is presented in Fig. 7. In order to limit statistical fluctuations, spatially-averaged values were calculated. Temperatures of the X-ray ejection were obtained in two ways: together with feature B when these structures were along the line of sight, and separately when the front of the X-ray ejection passed feature $\mathrm{B}$. This is the reason for a discontinuity of the "crossed" curve about 05:31 UT. After that time the separate temperatures for the front and for the interior of the X-ray ejection are plotted.

We see that any evident temperature increase in the NOAA AR 8210 before the main X1.2 flare was connected with the X-ray ejection. A strong jump is visible about 05:29 UT and two minutes later the plasma in the X-ray ejection reached the maximum temperature of $8.2 \mathrm{MK}$. We also see that the hot plasma was concentrated in the front of the X-ray ejection. The interior was evidently cooler. The temperature increase between 05:18-05:22 UT was probably caused by a formation of feature $B$ which remained static later on. Also feature A showed a stable temperature $(\sim 7.5 \mathrm{MK})$ until 05:32 UT, followed by a steeper drop. The temperature values from the ratio of SXT filters Be119 and Al12, available for a northern part of the X-ray ejection front, were in the range 11.1-8.4 MK for the period 05:30-05:36 UT.

To obtain other physical parameters of the X-ray ejection we estimated a lower and upper limit of the X-ray ejection volume assuming a hemishell and a hemisphere geometry, respectively. In the first case, the thickness of the hemishell was assumed to be equal to the distance between the leading and the trailing edge of the X-ray ejection front (Fig. 5). In the second case, the hemisphere radius was assumed to be equal to the actual height (above the chromosphere) of the leading edge of the X-ray ejection. In both cases we should remember that a fraction of the X-ray ejection was occulted by the solar limb. Both limits of the visible volume of the X-ray ejection, $V_{\text {vis }}^{\min }$ and $V_{\text {vis }}^{\max }$, as well as both limits of the total volume, $V_{\text {tot }}^{\min }$ 


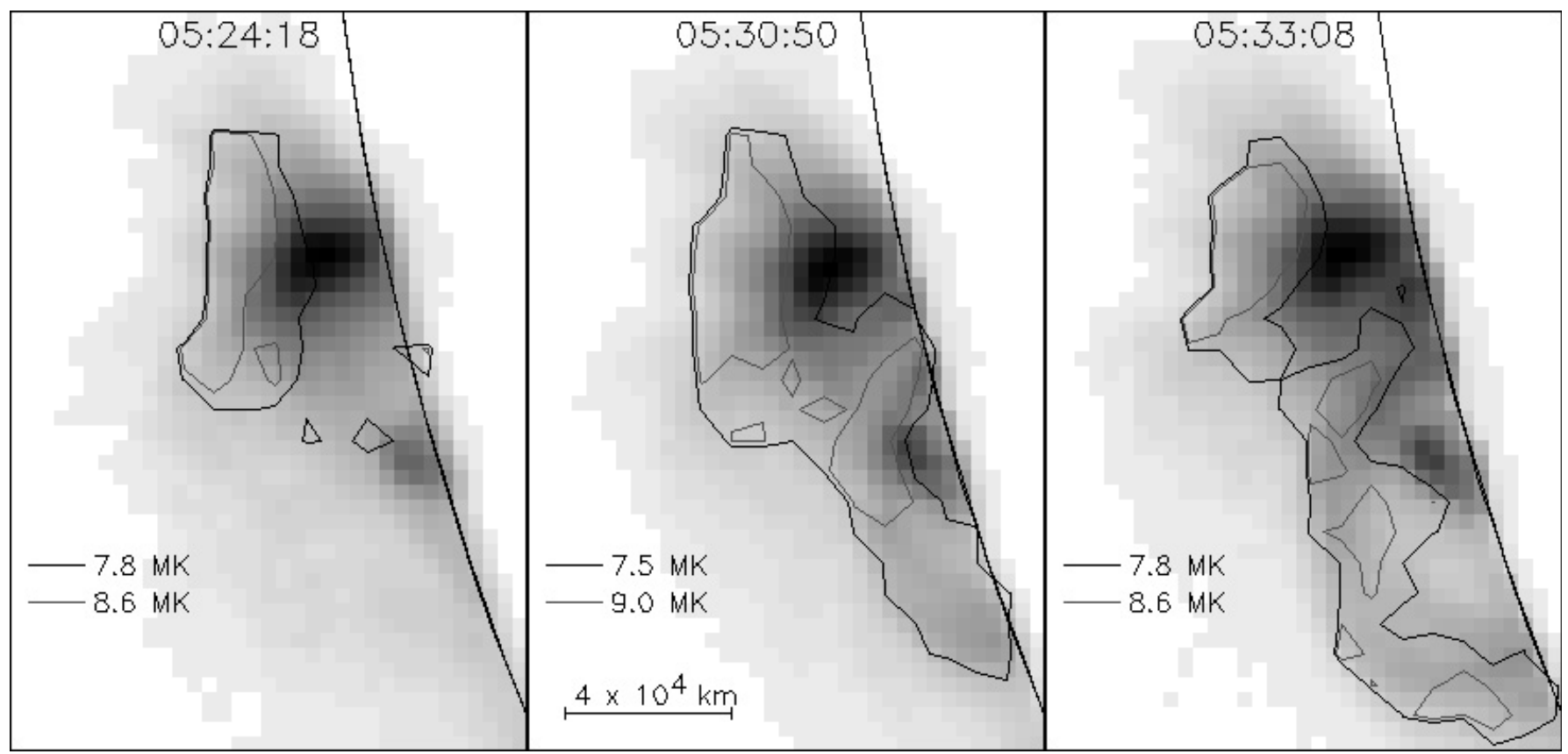

Fig. 6. An example of Al12/AlMg temperature maps of the NOAA AR 8210 before and during the X-ray ejection. Lines of constant temperature are overplotted on the SXT FN AlMg images. See the caption of Fig. 3 for more details.

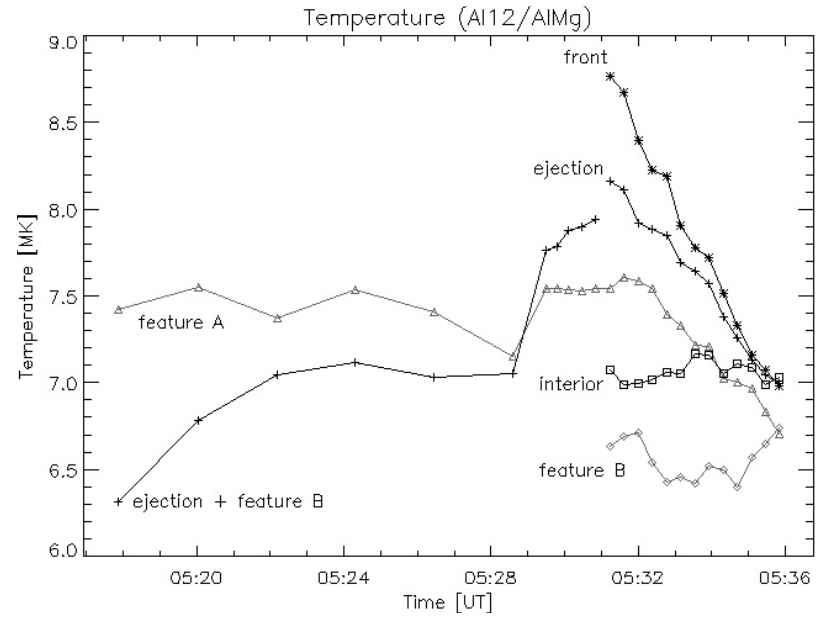

Fig. 7. The Al12/AlMg temperature changes for the selected parts of the NOAA AR 8210 before and during the X-ray ejection. See text for explanation.

and $V_{\text {tot }}^{\max }$, are listed in Table 1 together with temperature, $T$, and emission measure, $E M$.

The remaining parameters presented in Table 1 were calculated in the standard way:

- electron density, $n_{\mathrm{e}}=\left(E M / V_{\text {vis }}\right)^{1 / 2}$, where $E M$ is emission measure;

- electron pressure, $p_{\mathrm{e}}=n_{\mathrm{e}} k_{\mathrm{B}} T$, where $k_{\mathrm{B}}$ is the Boltzmann constant;

- mass, $M=n_{\mathrm{e}} m_{\mathrm{p}} V_{\mathrm{tot}}$, where $m_{\mathrm{p}}$ is the proton mass.

The calculated values of the above described parameters allow us to investigate a detailed energy balance of the X-ray ejection.
Following Webb et al. (1980) and Ohyama \& Shibata (1997) the following formula was used:

$\dot{E}_{\mathrm{rel}} \approx \dot{E}_{\mathrm{th}}+\dot{E}_{\mathrm{kin}}+\dot{E}_{\mathrm{g}}+\dot{E}_{\mathrm{rad}}+\dot{E}_{\mathrm{c}} \quad\left[\mathrm{erg} \mathrm{s}^{-1}\right]$

where $\dot{E}_{\text {rel }}$ is the rate of the energy release, $E_{\text {th }}=3 n_{\mathrm{e}} k_{\mathrm{B}} T V_{\text {tot }}$ is the total thermal energy content, $E_{\text {kin }}=0.5 M v_{\text {front }}^{2}$ is the kinetic energy ( $v_{\text {front }}-$ from Fig. 5), $\left|E_{\mathrm{g}}\right|=M g_{\odot} h_{\text {front }}$ is the gravitational energy $\left(g_{\odot}-\right.$ acceleration of solar surface gravity, $h_{\text {front }}-$ from Fig. 5), $\dot{E}_{\text {rad }}=n_{\mathrm{e}}^{2} \Lambda(T) V_{\text {tot }}$ is the energy loss rate due to radiation $(\Lambda(T)$ - the radiative loss function $\left.\simeq 4 \times 10^{-23} \mathrm{erg} \mathrm{s}^{-1} \mathrm{~cm}^{3}\right), \dot{E}_{\mathrm{c}}=\kappa_{0} T^{3.5} S L^{-1}$ is the energy loss rate due to conduction $\left(\kappa_{0}=10^{-6} \mathrm{erg} \mathrm{s}^{-1} \mathrm{~cm}^{-1} \mathrm{~K}^{-3.5}, S-\right.$ area of a contact with the chromosphere, $L$ - characteristic path of conduction $^{1}$ ). Energy rates, $\dot{E}_{\mathrm{th}}, \dot{E}_{\mathrm{kin}}$, and $\dot{E}_{\mathrm{g}}$, in Eq. (2) were calculated as follows:

$\dot{E}=\frac{E(t+\tau)-E(t)}{\tau}$

where $\tau$ is the time interval between two consecutive SXT images.

The time evolution of individual energy rates estimated for the X-ray ejection is plotted in Fig. 8. Each energy rate is represented by two curves which limit the most probable area of actual values. At the beginning of the X-ray ejection, most of the released energy is spent on increasing the thermal energy content. Later, the kinetic energy and the gravitational energy increase and become comparable with the thermal energy content. The energy loss rate due to radiation is omitted in Fig. 8 because of its marginal importance in the energy balance of the $\mathrm{X}$-ray ejection $\left(\$ 2 \times 10^{25} \mathrm{erg} \mathrm{s}^{-1}\right)$.

\footnotetext{
${ }^{1}$ It has been assumed that the $S / L$ ratio values were the same as for a loop having a radius and thickness equal to the radius and the thickness of the bright boundary of the X-ray ejection.
} 
Table 1. Values of parameters characterizing the X-ray ejection.

\begin{tabular}{|c|c|c|c|c|c|c|c|}
\hline UT & $\begin{array}{c}\text { Tempe- } \\
\text { rature, } T \\
{\left[10^{6} \mathrm{~K}\right]}\end{array}$ & $\begin{array}{c}\text { Emission } \\
\text { measure, } E M \\
{\left[10^{47} \mathrm{~cm}^{-3}\right]}\end{array}$ & $\begin{array}{c}\text { Visible } \\
\text { volume, } V_{\text {vis }} \\
{\left[10^{29} \mathrm{~cm}^{3}\right]}\end{array}$ & $\begin{array}{c}\text { Total } \\
\text { volume, } V_{\text {tot }} \\
{\left[10^{29} \mathrm{~cm}^{3}\right]}\end{array}$ & $\begin{array}{c}\text { Electron } \\
\text { density, } n_{\mathrm{e}} \\
{\left[10^{9} \mathrm{~cm}^{-3}\right]}\end{array}$ & 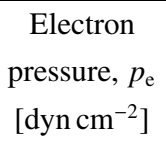 & $\begin{array}{l}\text { Mass, } M \\
{\left[10^{14} \mathrm{~g}\right]}\end{array}$ \\
\hline $05: 29: 28^{a}$ & 7.8 & 1.7 & $0.37-0.67$ & $0.78-1.1$ & $1.6-2.1$ & $1.7-2.3$ & $2.8-2.9$ \\
\hline $05: 29: 46^{a}$ & 7.8 & 2.0 & $0.37-0.69$ & $0.78-1.2$ & $1.7-2.3$ & $1.8-2.5$ & $3.0-3.3$ \\
\hline $05: 30: 06^{a}$ & 7.9 & 2.3 & $0.38-0.71$ & $0.78-1.2$ & $1.8-2.4$ & $1.9-2.7$ & $3.1-3.5$ \\
\hline $05: 30: 28^{a}$ & 7.9 & 2.6 & $0.40-0.76$ & $0.79-1.3$ & $1.8-2.6$ & $2.0-2.8$ & $3.3-3.9$ \\
\hline $05: 30: 50^{a}$ & 7.9 & 2.9 & $0.44-0.85$ & $0.84-1.4$ & $1.8-2.6$ & $2.0-2.8$ & $3.6-4.3$ \\
\hline $05: 31: 14$ & 8.2 & 2.7 & $0.51-0.99$ & $0.94-1.6$ & $1.7-2.3$ & $1.9-2.6$ & $3.6-4.4$ \\
\hline $05: 31: 36$ & 8.1 & 2.9 & $0.62-1.2$ & $1.1-1.9$ & $1.6-2.2$ & $1.8-2.4$ & $3.9-4.9$ \\
\hline 05:32:00 & 7.9 & 3.0 & $0.78-1.5$ & $1.3-2.3$ & $1.4-2.0$ & $1.6-2.2$ & $4.2-5.5$ \\
\hline $05: 32: 22$ & 7.9 & 3.3 & $0.99-1.8$ & $1.6-2.8$ & $1.4-1.8$ & $1.5-2.0$ & $4.8-6.3$ \\
\hline $05: 32: 46$ & 7.8 & 3.4 & $1.3-2.4$ & $2.0-3.5$ & $1.2-1.6$ & $1.3-1.7$ & $5.4-7.0$ \\
\hline 05:33:08 & 7.7 & 3.6 & $1.7-3.0$ & $2.6-4.4$ & $1.1-1.5$ & $1.2-1.5$ & $6.1-8.0$ \\
\hline 05:33:32 & 7.6 & 3.8 & $2.3-4.0$ & $3.3-5.6$ & $0.98-1.3$ & $1.0-1.3$ & $7.1-9.2$ \\
\hline 05:33:54 & 7.6 & 3.7 & $3.1-5.1$ & $4.3-7.1$ & $0.85-1.1$ & $0.88-1.1$ & $7.8-10.0$ \\
\hline $05: 34: 18$ & 7.4 & $3.8^{b}$ & $4.2-6.8$ & $5.7-9.2$ & $0.75-0.95$ & $0.76-0.97$ & $9.0-11.4$ \\
\hline $05: 34: 40$ & 7.3 & $3.6^{b}$ & $5.6-8.7$ & $7.3-11.6$ & $0.64-0.80$ & $0.64-0.80$ & $9.7-12.3$ \\
\hline 05:35:04 & 7.1 & $3.3^{b}$ & $7.5-11.5$ & $9.7-15.0$ & $0.53-0.66$ & $0.52-0.65$ & $10.6-13.3$ \\
\hline $05: 35: 26$ & 7.0 & $2.9^{b}$ & $9.9-14.8$ & $12.4-18.9$ & $0.45-0.55$ & $0.43-0.53$ & $11.3-14.0$ \\
\hline 05:35:50 & 7.0 & $2.6^{b}$ & $13.2-19.3$ & $16.2-24.2$ & $0.36-0.44$ & $0.35-0.43$ & $11.9-14.7$ \\
\hline
\end{tabular}

${ }^{a}$ The signal from feature B was included. Actual temperature values should be somewhat higher, whereas actual values of emission measure, electron density, electron pressure and mass should be somewhat lower.

${ }^{b}$ The signal from the part of the X-ray ejection that runs away outside the HN field of view was added from the SXT QN AlMg images (so-called Quarter Normal resolution, QN - pixel size 9!'8 × 9!'8).

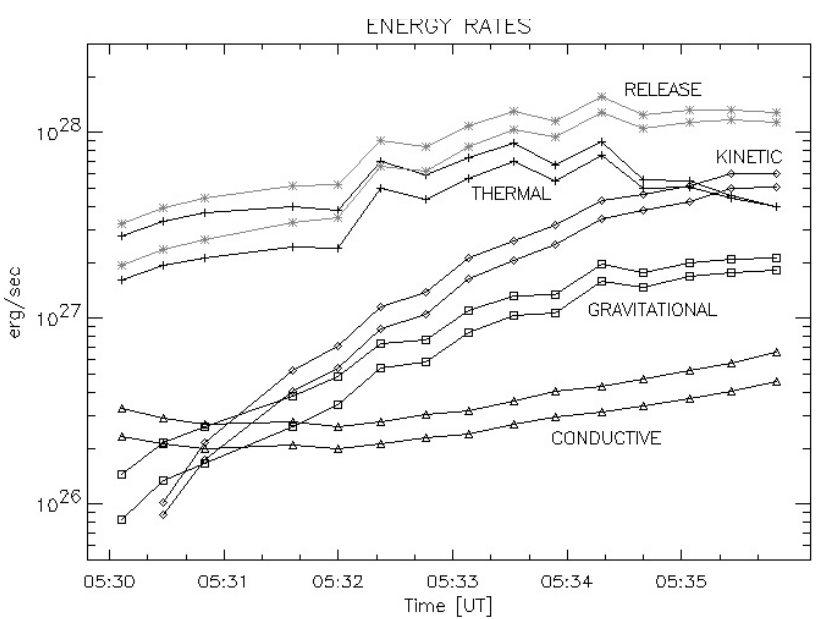

Fig. 8. The time evolution of individual energy rates estimated for the X-ray ejection. See text for explanation.

The total energy released to feed the X-ray ejection was estimated to be in the range $3-4 \times 10^{30}$ erg for the period 05:28-05:36 UT and 1-2 $\times 10^{31}$ erg for the whole duration (see Sect. 2.2). Comparing it to the typical total energy $\left(\sim 10^{32} \mathrm{erg}\right)$ for an X-class flare (e.g. Somov 1992), we see that this is a quite important quantity in the total energy budget of the flare.

\subsection{Coronal mass ejection}

A sequence of images of the CME which developed on the eastern solar limb on 23 April 1998 has been obtained by the Large Angle Spectroscopic Coronagraph (LASCO) onboard the Solar and Heliospheric Observatory (SOHO) mission. According to St. Cyr's CME list 1998 on the SOHO/LASCO Web site ${ }^{2}$ this CME started at 05:27 UT and was classified as a halo event. Running-difference images made by $\mathrm{C} 2$ and $\mathrm{C} 3$ coronagraphs illustrating the CME evolution are presented in Fig. 9. We see that the angular extension of this CME was about $100^{\circ}-120^{\circ}$ in the early phase of its evolution and the NOAA AR 8210 had a central situation in comparison with the CME.

Temporal comparison between the X-ray ejection and the CME proves that the X-ray could not initiate the CME. One minute before the first indication of the $\mathrm{X}$-ray ejection, the front of the CME was observed far above the solar surface, at a distance of about $2.8 R_{\odot}$.

Images made by the $\mathrm{LASCO} / \mathrm{C} 1$ coronagraph and by the Extreme Ultraviolet Imaging Telescope (EIT) also onboard SOHO show that some magnetic processes which preceded the CME formation began in the NOAA AR 8210 immediately after 04:00 UT. Running difference images presented in Fig. 10 show a progressive dimming in the NOAA AR 8210 which is commonly treated as the CME formation signature

\footnotetext{
${ }^{2}$ http://cdaw.gsfc.nasa.gov/CME_list
} 


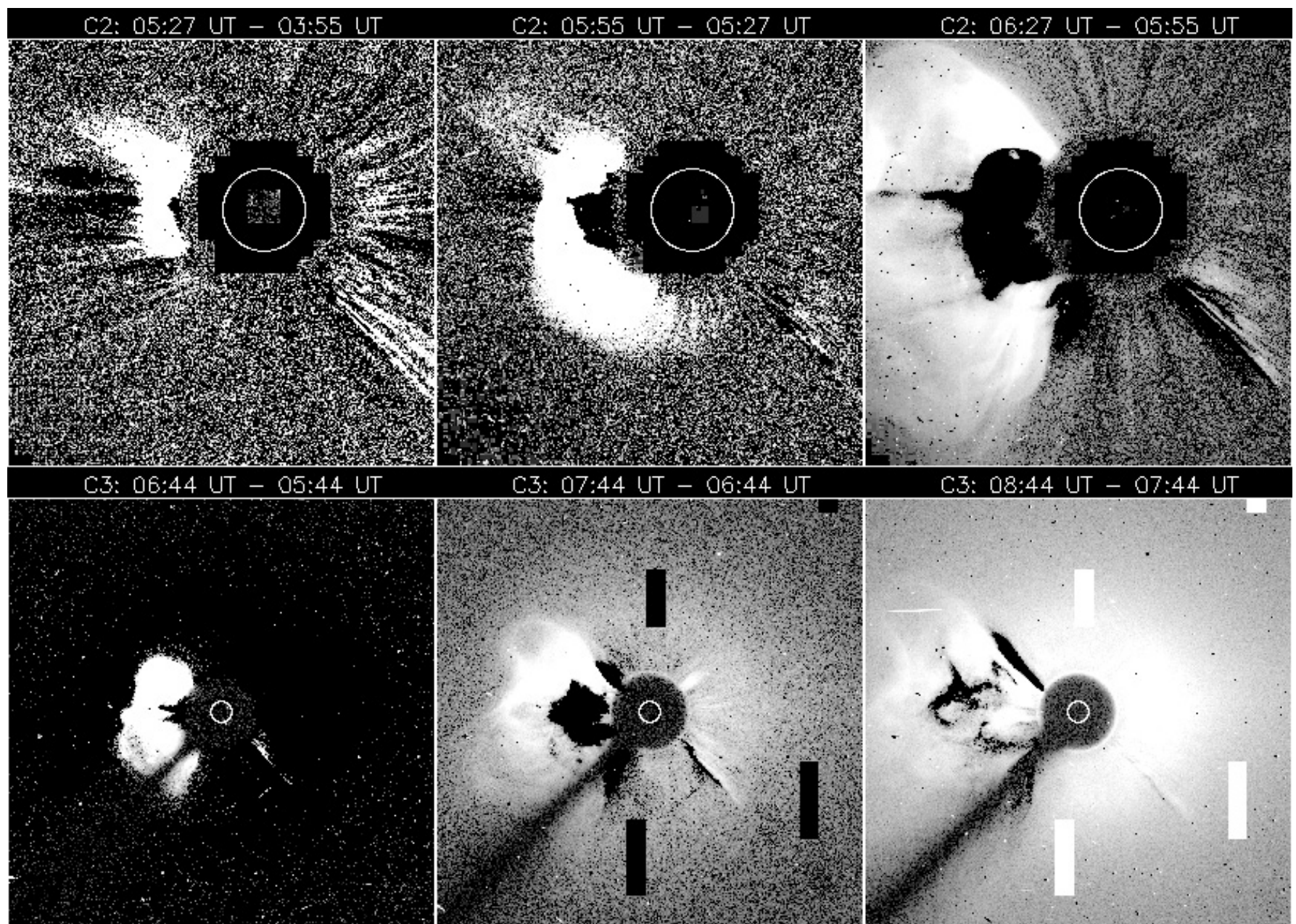

Fig. 9. Running-difference images of LASCO/C2 (upper three panels) and LASCO/C3 (lower three panels), which show the evolution of the halo CME on 23 April 1998. Time is indicated at the top of each panel. The white circle in each panel indicates the solar limb (1.0 $\left.R_{\odot}\right)$.

(e.g. Hudson et al. 1996). In the EIT $\lambda 195 \AA$ A (Fe XII) images the volume of emission depletion did not form a regular front, however, this may be caused by the relatively narrow field of view of this instrument. In the $\mathrm{LASCO} / \mathrm{C} 1$ images made in the green line of Fe XIV at $5303 \AA$ a regular bubble is seen which expanded both radially and laterally.

Figure 11 shows the variation of height with time (dashed line) of the CME observed with three LASCO coronagraphs. The height of the CME at a given snapshot image is defined by radial distance of the leading edge along the central direction. The uncertainty of the CME height is estimated to be about $0.01,0.1$, and $0.5 R_{\odot}$ for $\mathrm{C} 1, \mathrm{C} 2$, and $\mathrm{C} 3$, respectively. In the images made at 05:14 UT and 06:27 UT the CME front has moved outside the instrument field of view, thus the symbols denote then the lower approximation of the height.

The variation of the CME speed in the radial direction is presented in Fig. 12. The velocity is derived by using the height difference between the images at the given time and the previous image, thus, it is the average velocity during the period between two adjacent observations. Since the CME undergoes fast velocity changes and due to a lack of a sufficient number of measurements, the polynomial fitting method is not applicable here. The uncertainty of velocity is caused by the uncertainty in measuring the CME height.
Figure 12 shows that the CME underwent two phases of a rapid acceleration. The first phase occurred somewhere between 04:51-05:27 UT, the second one somewhere between 05:55-06:44 UT. The average acceleration was estimated to be about $0.17 \mathrm{~km} \mathrm{~s}^{-2}$, and $0.14 \mathrm{~km} \mathrm{~s}^{-2}$ for the first and the second phase, respectively. Because of the poor time sampling of the LASCO observations these values are probably underestimated.

\section{Discussion}

Zhang et al. (2001) found that the kinetic evolution of flareassociated CMEs can be described in a three-phase scenario: the initiation phase, impulsive acceleration phase, and propagation phase. The CMEs are initiated at the height of $1.3-1.5 R_{\odot}$ and accelerated up to the height of 3.7-4.7 $R_{\odot}$. In the impulsive acceleration phase the average acceleration is in the range of $100-500 \mathrm{~m} \mathrm{~s}^{-2}$.

The velocity-time profile of the investigated CME (Fig. 12) shows a more complex appearance. It can be divided into: the initiation phase before 04:51 UT, the first impulsive acceleration phase between 04:51-05:27 UT, the first propagation phase between 05:27-05:55 UT, a second impulsive acceleration phase between 05:55-06:44 UT, and a second propagation phase after 06:44 UT. The impulsive acceleration phases 


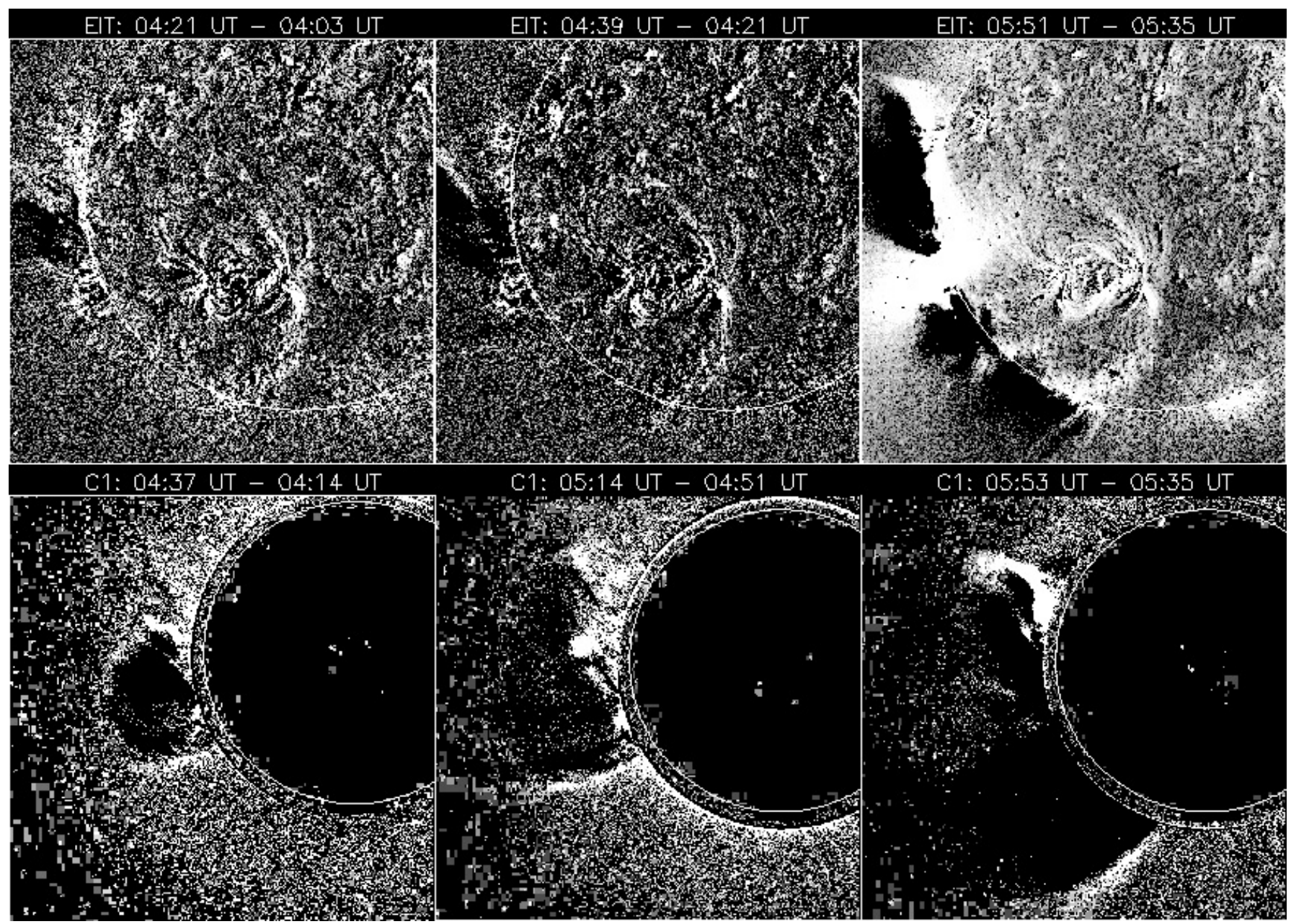

Fig. 10. Running-difference images of EIT $\lambda 195 \AA$ (upper three panels) and LASCO/C1 $\lambda 5303 \AA$ (lower three panels), which show the early evolution of the halo CME on 23 April 1998. Time is indicated at the top of each panel. The white circle in each panel indicates the solar limb $\left(1.0 R_{\odot}\right)$.

occurred when the CME front heights were somewhere between $1.7-3.1 R_{\odot}$, and between $4.5-9.0 R_{\odot}$ for the first and second phase, respectively.

We can speculate that, if there were no second impulsive acceleration phase, the CME on 23 April 1998 would be classified as non-flare-associated due to its moderate velocity below $600 \mathrm{~km} \mathrm{~s}^{-1}$ after the first impulsive acceleration phase and the lack of association with any flare (see Fig. 1). The CME reached a velocity of about $1000 \mathrm{~km} \mathrm{~s}^{-1}$ thanks to the second impulsive acceleration phase which occurred unusually high in the corona in comparison with the results obtained by Zhang et al. (2001) for other flare-associated events.

We will show that the second impulsive acceleration phase of the CME could have been caused by the X-ray ejection associated with the X1.2-flare. In Fig. 11, the position of the CME front after the first impulsive acceleration phase is extrapolated (straight solid lines) assuming a constant velocity of $580 \pm 40 \mathrm{~km} \mathrm{~s}^{-1}$, the same as that obtained from the position changes of the CME front between LASCO/C2 images taken at 05:27 and 05:55 UT. Moreover, the position of the X-ray ejection front was extrapolated (parabolic curves) assuming constant accelerations of $0.95 \mathrm{~km} \mathrm{~s}^{-2}$ and $0.44 \mathrm{~km} \mathrm{~s}^{-2}$ for the leading and the trailing edge, respectively. These values were obtained from a least-square polynomial fit to the SXT data (Fig. 5).

The intersection of these two extrapolated curves in Fig. 11, which can be interpreted as a collision of the X-ray ejection and the CME front, occurred at the height of $(5.3 \pm 0.1) R_{\odot}$ at about 06:10-06:12 UT; i.e. this occurred during the second impulsive acceleration phase of the CME (Fig. 12). Thus, a hypothesis that the X-ray ejection caused the second impulsive acceleration phase should be seriously considered.

This hypothesis is to some extend supported by other independent observations, such as the metric type II radio bursts detected with the radio spectrograph of the Astrophysikalisches Institut Potsdam. It has been generally accepted that this kind of radiation is a radio signature of coronal MHD-shock waves (Uchida 1960). Recently some X-ray ejections have been identified as the drivers of coronal shock waves (Gopalswamy et al. 1997; Klein et al. 1999; Gopalswamy et al. 2001).

According to the event catalog ${ }^{3}$, type II radio bursts were observed at the frequency range between $170-40 \mathrm{MHz}$

http://www.aip.de/People/AKlassen 


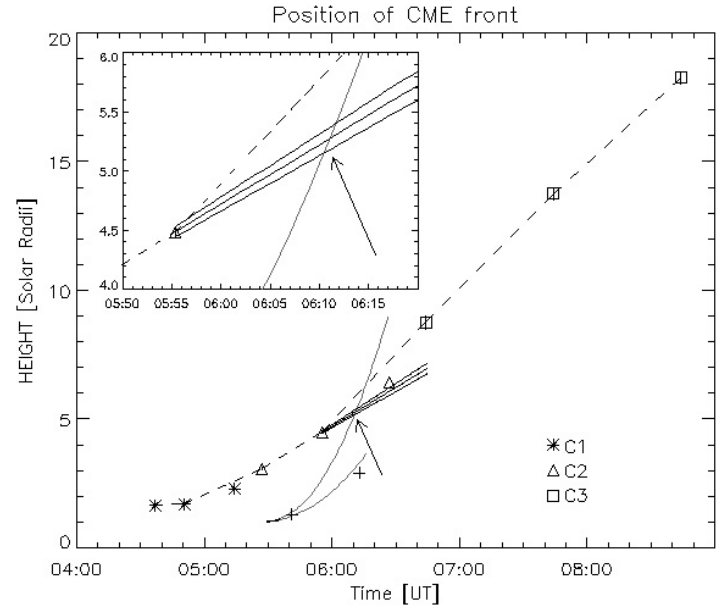

Fig. 11. Height-time plot (dashed line) of the CME front on 23 April 1998. The CME heights were measured in LASCO images. Different symbols: asterisks, triangles, and squares denote $\mathrm{C} 1$, $\mathrm{C} 2$, and $\mathrm{C} 3$ images, respectively. The parabolas represent the position of the leading and the trailing edge of the $\mathrm{X}$-ray ejection observed (Fig. 5) and extrapolated under assumption of their constant acceleration $0.95 \mathrm{~km} \mathrm{~s}^{-2}$, and $0.44 \mathrm{~km} \mathrm{~s}^{-2}$, respectively. Three straight lines coming out from the point at 05:55 UT represent an extrapolated position of the CME front under assumption of the constant velocity 540, 580 , and $620 \mathrm{~km} \mathrm{~s}^{-1}$, respectively. The estimated height of the X-ray ejection front from type II radio bursts is marked with crosses. The suggested place of the collision between the fronts of the CME and the X-ray ejection is marked by the arrow and additionally blown-up in the insert (upper left corner).

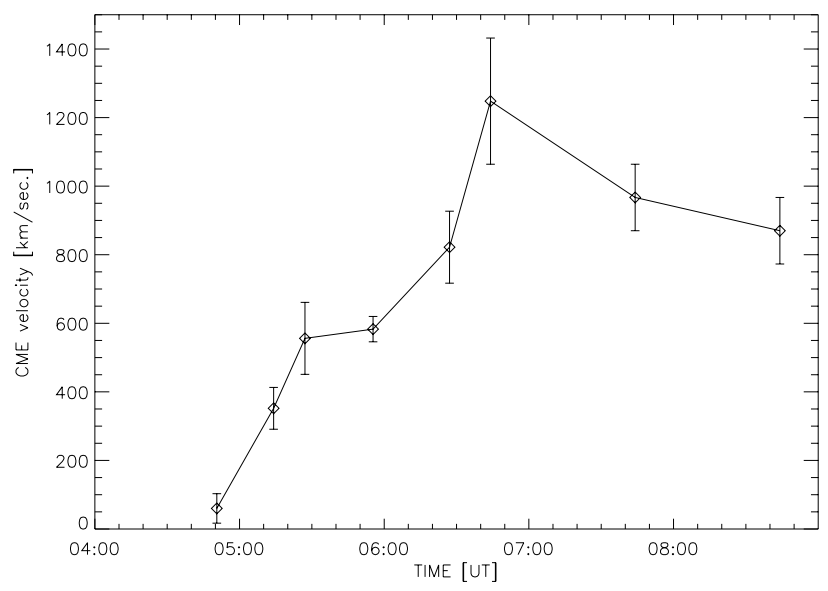

Fig. 12. Velocity-time profile of the CME front on 23 April 1998. Two phases of impulsive acceleration are seen: the first somewhere between 04:51-05:27 UT, the second somewhere between 05:55-06:44 UT.

starting from 05:41 UT until 06:13 UT. Both fundamental and harmonic emission bands were seen.

The observed frequency in a dynamic radio spectrum can be related to a position in the solar corona as long as the density distribution in the corona is known. Following Classen \& Aurass (2002), a four-fold Newkirk (1961) model was assumed. On this basis, the height of MHD-shock waves was estimated. Their leading edge was at about $1.3 R_{\odot}$ at 05:41 UT (the fundamental emission at $170 \mathrm{MHz}$ ) and their trailing edge was at about $2.9 R_{\odot}$ at 06:13 UT (the harmonic emission at $40 \mathrm{MHz}$ ) - see the crosses in Fig. 11. (At 06:13 UT the leading edge of the MHD shock wave was far outside the frequency range monitored by the Potsdam radio spectrograph.) The position of MHD-shock waves agrees very well with the extrapolated position of the X-ray ejection front. In this way we obtained an independent proof that the X-ray ejection front did not change its acceleration after it faded in the SXT images.

The postulated collision between the X-ray ejection front and the CME front can be used for an independent estimation of the CME mass. In the calculation we took the mass of the X-ray ejection determined from SXT data, 1.2-1.5 $\times$ $10^{15} \mathrm{~g}$ (Table 1). We used the following values of velocity: $2350 \mathrm{~km} \mathrm{~s}^{-1}, 580 \mathrm{~km} \mathrm{~s}^{-1}$, and $1000 \mathrm{~km} \mathrm{~s}^{-1}$ for the X-ray ejection front before the collision, for the CME front before the collision, and for the CME front after the collision, respectively. The first value was extrapolated under the assumption of a constant acceleration of $0.95 \mathrm{~km} \mathrm{~s}^{-2}$. The last two values were taken from Fig. 12. Assuming momentum conservation we determined the CME mass to be in the range 3.8-4.8 $\times$ $10^{15} \mathrm{~g}$ and the total mass of the X-ray ejection and the CME to be in the range $5.0-6.3 \times 10^{15} \mathrm{~g}$. The obtained values agree very well with other estimations of CME masses (e.g. Vourlidas et al. 2002).

\section{A possible scenario of the event}

A magnetic process leading to the opening of previously closed field lines along a zero line of the longitudinal magnetic field (Švestka 2001) started on 23 April 1998 in the NOAA AR 8210 immediately after 04:00 UT. The signatures of such a process are: the increase in the GOES light curves (Fig. 1) and the dimming observed in the EIT $\lambda 195 \AA$ image (see upper, left panel in Fig. 10). A trigger which destabilized the magnetic structure might be an emerging magnetic flux. The evidence of such a process was seen many times during the passage of the NOAA AR 8210 across the solar disc (e.g. Kołomański \& Jakimiec 2002).

The CME was formed not later than at 04:37 UT with a front at a height of about $1.7 R_{\odot}$ (see bottom, left panel in Fig. 10). The time interval of a slow expansion of the CME lasted at least until 04:51 UT. The next LASCO/C1 image (bottom, middle panel in Fig. 10) shows an evident increase of the expansion rate. The CME expanded both radially and laterally. By comparing with the LASCO/C2 image (upper, left panel in Fig. 9) it is possible to establish a time interval of strong acceleration of the CME. This lasted between 04:51-05:27 UT, no longer than $36 \mathrm{~min}$. After this phase the CME front reached a height of $3.1 R_{\odot}$ and expanded with approximately constant velocity of about $580 \mathrm{~km} \mathrm{~s}^{-1}$.

Such a development, including a low formation height, impulsive and strong acceleration and a high speed relatively close to the solar surface, is typical for flare-associated CMEs. On the other hand, no flare was detected before 05:32 UT (see Fig. 1). We will try to explain this discrepancy later.

A further evolution of the magnetic field in the NOAA AR 8210 caused the flare. The reconnection of magnetic lines began no later than 05:28 UT, because from this 
moment the signatures of hot plasma became visible (Fig. 2). If we extrapolate the height-time plot for the X-ray ejection (Fig. 5), we obtain a reconnection point at a height of about $3 \times$ $10^{4} \mathrm{~km}$, i.e. above the solar limb. However, another possibility is that the reconnection occurred behind the solar limb (i.e. much closer to the solar surface), and at 05:28 UT the X-ray ejection became visible above the solar limb. In this case the reconnection occurred several minutes earlier.

The consequence of the reconnection process was the X-ray ejection propagating upward from the reconnection point (e.g. Yokoyama \& Shibata 2001). Below the reconnection point a sudden development of MHD turbulence occurred, in which a large amount of plasma was efficiently heated and a huge number of electrons was accelerated to high energies (Jakimiec et al. 1998).

The X-ray ejection took the form of a rapidly expanding system of magnetic loops. At the early stage of evolution the hot plasma was found in the X-ray ejection front (Figs. 2 and 6). The X-ray ejection expanded with a constant acceleration, larger for the leading edge of the front and smaller for the trailing edge, $0.95 \mathrm{~km} \mathrm{~s}^{-2}$ and $0.44 \mathrm{~km} \mathrm{~s}^{-2}$, respectively (Fig. 5).

The top of the flaring arcade came into view above the solar limb at 05:37 UT (Fig. 3). Thanks to the specific configuration, the flare emission did not dominate the emission of the X-ray ejection. Consequently, this allowed us to identify the X-ray ejection as the precursor of the main flare (Fig. 4).

After 05:36 UT, when the X-ray ejection front faded and left the field of view of the SXT telescope, it seems that the $\mathrm{X}$-ray ejection still expanded with the same acceleration. This suggestion is confirmed by the type II radio burst observations (Fig. 11). If this interpretation is correct it means that in our event coronal MHD-shock waves were driven by the X-ray ejection as piston. Thus, this is an example of the class 3 of metric type II burst excitation in the classification of Classen \& Aurass (2002).

A further expansion with the same acceleration should result in a collision of the X-ray ejection front with a much slower CME front. Such an interaction probably happened, because between the LASCO/C2 images at 05:55 UT and 06:27 UT (Fig. 9) the expansion of the CME front evidently accelerated. In Fig. 11 the extrapolations of the positions of the X-ray ejection front and the CME front intersect each other at about 06:11 UT. After 06:44 UT the CME front velocity did not increase. On the contrary, some signatures of a deceleration were seen (Fig. 12).

Estimates of the X-ray ejection mass, $1.2-1.5 \times 10^{15} \mathrm{~g}$, and of the total energy released to drive the $\mathrm{X}$-ray ejection, $1-2 \times$ $10^{31} \mathrm{erg}$, prove that this ejection was sufficiently massive and energetic to cause the observed acceleration of the CME front. Also the CME mass estimate obtained under the assumption of momentum conservation seems to be acceptable.

What were the reasons for the impulsive acceleration of the CME between 04:51 and 05:27 UT, when no flare occurred? We suggest that a reconnection process in the NOAA AR 8210 began before 05:28 UT, when the flare started. Recurrent signatures of magnetic activity in the low corona are seen in the EIT and LASCO/C1 images (Fig. 10) made between the CME formation (not later than 04:37 UT) and the occurrence of X-ray ejection (05:28 UT).

Moreover, cusp-like details above the feature A are seen in Fig. 3. After the X-ray ejection expanded, their number increased from 3 to 4 . If they were formed in the same way as the latest cusp, this means that at least three other X-ray ejections were formed before the ejection which started at 05:28 UT. They could accelerate the CME front in the same way as we described earlier. Unfortunately, the propagation of earlier X-ray ejections was not observed by Yohkoh due to the satellite night.

One flare can produce more X-ray ejections at different times and locations (Nitta \& Akiyama 1999). Some features observed during the evolution of the X1.2 flare investigated in this paper can also be interpreted as signatures of further $\mathrm{X}$-ray ejections, e.g., the hard X-ray source E recorded by Sato (2001).

If such analogies can justify the hypothesis of the occurrence of many X-ray ejections in the NOAA AR 8210 on 23 April 1998, it means that this active region probably underwent a stage of quasi-stationary evolution before reaching a critical point at which violent magnetic activity caused the flare.

\section{Conclusions}

In this paper the analysis of a complex event, including a CME, an X-ray ejection, and a flare, has been presented. This event occurred in the NOAA AR 8210 on 23 April 1998. The observations from Yohkoh and SOHO (LASCO and EIT) have been used. Basic conclusions can be summarized as follows:

1. The X-ray ejections associated with behind-the-limb flares can be analysed with better quality than the events for which the emission of X-ray ejections is dominated by flare emission.

2. In the soft X-ray light curves, the X-ray ejection is the precursor of the main flare.

3. The X-ray ejection front contains a very hot thermal plasma.

4. The X-ray ejection is responsible for an additional acceleration of the CME.

5. An interaction between the X-ray ejection and the CME has been used to develop a new method of the CME mass estimation.

The hypothesis that the X-ray ejections are responsible for an additional acceleration of the CMEs looks exciting, however, this is only a single example and a confirmation for more events is needed.

Acknowledgements. The Yohkoh satellite is a project of the Institute of Space and Astronautical Science of Japan. The $\mathrm{SOHO}$ project is a joint international space mission carried out by the ESA and NASA. I thank Professor J. Jakimiec and Dr. P. Preś for many useful comments and discussions. I also thank Dr. A. Fludra for comments on the manuscript and the anonymous referee for valuable remarks which led to substantial improvement of this paper. This work was supported by the Polish Committee for Scientific Research (KBN) grant No. 2 P03D 00123. 


\section{References}

Andrews, M. D., \& Howard, R. S. 2001, Space Sci. Rev., 95, 147

Classen, H. T., \& Aurass, H. 2002, A\&A, 384, 1098

Gopalswamy, N., Kundu, M. R., Manoharan, P. K, et al. 1997, ApJ, 486, 1036

Gopalswamy, N., St. Cyr, O. C., Kaiser, M. L., \& Yashiro, S. 2001, Sol. Phys., 203, 149

Harrison, R. A. 1986, A\&A, 162, 283

Hudson, H. S., Acton, L. W., \& Freeland, S. L. 1996, ApJ, 470, 629

Hudson, H. S., Kosugi, T., Nitta, N. V., \& Shimojo, M. 2001, ApJ, 561, L211

Jakimiec, J., Tomczak, M., Falewicz, R., Phillips, K. J. H., \& Fludra, A. 1998, A\&A, 334, 1112

Kahler, S. H. 1992, ARA\&A, 30, 113

Klein, K.-L., Khan, J. I., Vilmer, N., Delouis, J.-M., \& Aurass, H. 1999, A\&A, 346, L53

Kołomański, S., \& Jakimiec, J. 2002, ESA SP-506, 665

Kundu, M. R., Nindos, A., Vilmer, N., et al. 2001, ApJ, 559, 443

MacQueen, R. M., \& Fisher, R. R. 1983, Sol. Phys., 89, 89

Moon, Y.-J., Choe, G. S., Wang, H., et al. 2002, ApJ, 581, 694

Newkirk, G. Jr. 1961, ApJ, 133, 983

Nitta, N., \& Akiyama, S. 1999, ApJ, 525, L57
Ohyama, M., \& Shibata, K. 1997, PASJ, 49, 249

Ohyama, M., \& Shibata, K. 1998, ApJ, 499, 934

Sato, J. 2001, ApJ, 558, L37

Sheeley, N. R. Jr., Walters, H., Wang, Y.-M., \& Howard, R. A. 1999, JGR, 104, 24739

Shibata, K., Masuda, S., Shimojo, M., et al. 1995, ApJ, 451, L83

Somov, B. V. 1992, Physical Processes in Solar Flares (Dordrecht: Kluwer Acad. Publ.)

St.Cyr, O. C., et al. 2000, JGR, 105, 18169

Švestka, Z. 2001, Space Sci. Rev., 95, 135

Tsuneta, S. 1997, ApJ, 483, 507

Uchida, Y. 1960, PASJ, 12, 376

Vourlidas, O., Buzasi, D., \& Howard, R. 2002, ESA SP-506, 91

Webb, D. F., Cheng, C. C., Dulk, G. A., et al. 1980, in Solar Flares, a Monograph from Skylab Solar Workshop II, ed. P. A. Sturrock (Colorado Associated University Press), 471

Wu, S. T., Andrews, M. D., \& Plunkett, S. P. 2001, Space Sci. Rev., 95, 191

Yokoyama, T., \& Shibata, K. 2001, ApJ, 549, 1160

Zhang, J., Dere, K. P., Howard, R. A., Kundu, M. R., \& White, S. M. 2001, ApJ, 559, 452 